\title{
Three Harm-Based Arguments for a Moral Obligation to Vaccinate
}

\author{
Viktor Ivanković ${ }^{1} \mathbb{D} \cdot$ Lovro Savić $^{2}$
}

Accepted: 13 October 2021 / Published online: 5 November 2021

(c) Springer Science+Business Media, LLC, part of Springer Nature 2021

\begin{abstract}
A particularly strong reason to vaccinate against transmittable diseases, based on considerations of harm, is to contribute to the realization of population-level herd immunity. We argue, however, that herd immunity alone is insufficient for deriving a strong harm-based moral obligation to vaccinate in all circumstances, since the obligation significantly weakens well above and well below the herd immunity threshold. The paper offers two additional harm-based arguments that, together with the herd immunity argument, consolidates our moral obligation. First, we argue that individuals should themselves aim not to expose others to risk of harm, and that this consideration becomes stronger the more non-vaccinated people there are, i.e., the further we are below herd immunity. Second, we elaborate on two pragmatic reasons to vaccinate beyond the realization of herd immunity, pertaining to instability of vaccination rates and population heterogeneity, and argue that vaccinating above the threshold should serve as a precautionary measure for buttressing herd immunity. We also show that considerations of harm have normative primacy in establishing this obligation over considerations of fairness. Although perfectly sound, considerations of fairness are, at worst secondary, or at best complementary to considerations of harm.
\end{abstract}

Keywords Harm · Herd immunity · Infectiousness · Precaution · Thresholds

Lovro Savić

lovro.savic@ethox.ox.ac.uk

1 Institute of Philosophy, Ulica grada Vukovara 54, 10000 Zagreb, Croatia

2 Ethox Centre, Wellcome Centre for Ethics and Humanities, and Green Templeton College, University of Oxford, Big Data Institute, Li Ka Shing Centre for Health Information and Discovery, Old Road Campus, Oxford OX3 7LF, United Kingdom 


\section{Introduction}

Owing to a considerable drop in the vaccination coverage of children (34\% over the span of 5 years), the Dubrovnik-Neretva county in southern Croatia suffered a significant outbreak of measles in 2018 [31]. For Dubrovnik, the county seat, a small city of roughly 40,000 citizens, with over 2200 unvaccinated children, such a development was alarming for future public health estimates. For herd immunity to be re-established, levels of vaccination coverage needed to rise dramatically. Yet, with levels at an alltime low, many parents in the city of Dubrovnik might have suggested, at the moment of outbreak, that their obligation to vaccinate their children was reduced in strength, now that the population was so far from realizing herd immunity. Their individual contributions to collective immunization lacked significance for the prevention of future outbreaks, the objection goes, seeing that few other parents were cooperating and the general population was so far from the herd immunity threshold.

In the ethical debate on vaccinating against transmittable diseases, considerations of harm are commonly expressed through the notion of herd immunity (e.g., [12, 29]). Although herd immunity is an invaluable method for preventing and reducing harm at the population level, it alone remains insufficient for deriving a strong moral obligation to vaccinate against transmittable diseases. This paper offers two additional harm-based arguments, which show our individual obligations to vaccinate to be strong even when the population is well below a herd immunity threshold, or well above it.

In the first section, we unpack the notion of herd immunity and the way in which it determines the varying strengths of individual obligations, given the changing levels of vaccination coverage. In the second section, we ground a strong obligation well below a herd immunity threshold by arguing that individuals should themselves aim not to expose others to risks of harm. This consideration becomes stronger, we claim, the lower vaccination coverage is, because there will consequently be more people to expose to risk of infection. The third section establishes the strength of the obligation well above the herd immunity threshold by exploring two pragmatic reasons to vaccinate-instability of vaccination rates and population heterogeneity. These circumstances are normatively important because of their tendency to obtain in virtually any modern social setting. By combining the three harm-based arguments, the paper systematizes and further advances harm-based accounts in favor of an individual obligation to vaccinate. Moreover, we claim that harm-based considerations hold a normative primacy in establishing this moral obligation. There have been recent proposals that ground this obligation in considerations of fairness. Although we do not think the fairness account is unsound, we believe it is only secondary at best, given the strength of harm-based considerations.

\section{The Obligation to Realize Herd Immunity}

Infectious diseases can cause significant harms in populations. For example, measles can spread quickly, infecting as many as $90 \%$ of those who come into contact with the disease [3], and killing one person in every 5000 cases in high-income countries 
and as many as one person in every 100 cases in low-income countries [25]. Smallpox, marked by a $30 \%$ mortality rate, easy transmission, and no known treatment, is an even deadlier disease [13]. Influenza infects between 10 and $20 \%$ of the population during an average epidemic, and between 40 and $50 \%$ of schoolchildren and nursing home residents, to whom it is particularly dangerous [11: 411]. Other diseases-diphtheria, mumps, hepatitis A and B, typhoid, varicella, rubella, pertussis, polio, and now COVID-19-may also represent threats to public health. ${ }^{1}$

Individuals are not only faced with the threat of deadly harm, but due to the infectiousness of these diseases, they may facilitate the harm further. Battin et al. argue that individuals are vectors, and not just victims of infection, "embedded in a web of biological relationships" [4: 77]. Being a carrier of dangerous pathogens will in most cases introduce moral obligations for individuals, within constraints of demandingness and feasibility, to make sure not to spread them, often at the cost of their own preferences. Ignoring these obligations will often amount to "assaulting" those around us [4: 86]. Obligations may be borne by potential carriers as well; some diseases will spread quickly, and individuals will become asymptomatic carriers well before having a chance to know it. Admittedly, we may sometimes come to accept exposing others to risk at the social level. For instance, there is an overwhelming consensus in virtually all countries that we should not give up on driving simply because it carries significant potential to cause people harm. This is because driving produces significant benefits for everyone [20]. Risks of serious, and even deadly harm may thus be found acceptable, particularly when they are outweighed by significant collective benefits, although it may often be difficult to calculate between such risks and benefits. However, risks of harm from infectious diseases, as we claim in this paper, have hardly seemed acceptable since the emergence of vaccination. According to Jeffrey Ulmer and Margaret Liu, vaccines stand out as "the most efficacious and cost-effective of global medical interventions" [32: 291]. It is owing to vaccination campaigns that once common diseases, like diphtheria, have largely disappeared from public consciousness [12: 164], while some, like smallpox, have even been eradicated. ${ }^{2}$ In the great majority of cases, vaccinations are effective and safe, and most people take only minor risks to their health when they are administered. This is not to suggest that adverse effects from vaccines are never serious - the influenza vaccine, for instance, could cause Guillain-Barré Syndrome, which may lead to paralysis [6]. The chances, however, of suffering serious adverse effects are, in most cases and for most individuals, considerably smaller than risking the contraction of the infectious disease. Therefore, the choices of both vaccination

\footnotetext{
1 The better part of this article was written during the first months of the COVID-19 pandemic. We believe that any conclusions that we draw about moral obligations to vaccinate against the transmittable diseases mentioned here should apply in the case of COVID-19, now that effective and safe vaccines have been developed.

2 Vaccination programs standardly include the MMR (measles, mumps, rubella) vaccine, as well as vaccines against pneumococcal and meningococcal infections, hepatitis B, varicella, and others (see [10]).
} 
and non-vaccination come with a risk of harm to the choice-maker, but a prudent gambler would invariably bet on the vaccination. ${ }^{3}$

A small minority of individuals, however, would be prudent not to bet on vaccines. Public health experts identify three groups of persons who cannot be vaccinated for medical reasons, and are thus vulnerable to infectious diseases: (1) children too young to be vaccinated (e.g., children should not undergo vaccination against measles before the age of one [7]); (2) persons for whom the vaccination is ineffective (normally identified as vulnerable only after showing signs of infection); (3) persons who are immunosuppressed or seriously allergic to particular vaccines (see also [29: 387, 17: 548]). For members of these groups, the costs of vaccination are significantly higher.

Luckily, and most importantly for considerations of harm, these groups can be protected by indirect effects produced when others undergo vaccination. Successful vaccination reduces the transmission of infectious pathogens within the population, making it less likely that the vulnerable will be infected. The extent to which vaccination can produce indirect effects will depend on the particular infectious disease- how transmittable the pathogen is, or the nature and duration of the immunity induced by vaccination [15: 912] — but estimations can be made of vaccination rates to be reached in the population for the spread of a disease to be minimized, and for the non-vaccinated to be protected (e.g., for measles, the rate is 92-94\%). This threshold theorem is often referred to as 'herd immunity'. Assuming a "homogenously mixed" population ([1: 641, 15: 911]), reaching a herd immunity threshold entails that the vaccinated will "serve as a protective barrier against the likelihood of transmission" [22: 264], thus also preventing outbreaks of infection. Importantly, herd immunity is not a guarantee against the infection spreading altogether, but makes it much less likely that any infected individual will come into contact with those who are vulnerable [12: 162]. ${ }^{4}$

As a public good, herd immunity is both non-excludable and non-rivalrous. If a good is non-excludable, then no one can be excluded from the benefit produced by the good. All members of vulnerable groups will be afforded protection within a population that has realized herd immunity, but so will those who elude vaccination for non-medical reasons. The claim that herd immunity is non-rivalrous means that the extent to which a vulnerable (or any other) person benefits from herd immunity is not affected by the extent to which another person benefits from it ([12: 163-164, 17: 548]).

We join theorists who hold that we have a collective harm-based moral obligation to realize herd immunity (e.g., $[12,16,28])$. For individuals, this translates into a

\footnotetext{
3 To illustrate, the chances of suffering adverse effects from receiving a dozen standard vaccines for infectious diseases would still be decidedly slimmer than contracting the measles virus in a susceptible population. For those individuals unlucky enough to suffer adverse effects from vaccination, many countries have instituted injury compensation programs. See [21] for an overview of such programs.

4 Yash Paul has argued, and Dawson followed suit, that 'herd protection' is the more appropriate term for the described effect, while 'herd immunity' should denote the "secondary spread of attenuated viruses and bacteria" [27: 301]. For terminological consistency with the literature, we will stick to the more established term.
} 
personal obligation to undergo vaccination (or to have one's children vaccinated), when this does not incur significant medical costs to them (or their children). ${ }^{5}$ By doing so, the individual contributes to the collective effect of minimizing the risk of harm to members of all vulnerable groups. Conversely, the more individuals fail to vaccinate when herd immunity has been realized, the more likely it is that the good will be eroded, and that individuals (particularly those from vulnerable groups) will be susceptible to and suffer significant harms. ${ }^{6}$

Vulnerable individuals are normally identified as belonging to one of the groups we mentioned earlier. But realizing herd immunity also carries special significance in precarious circumstances for public health-e.g., when infections threaten public life (due to the seasonality of certain diseases), or when outbreaks erupt unexpectedly and are difficult to control [33: 3123]. Herd immunity will protect anyone in such circumstances who failed to protect herself via vaccination or who is underimmunized. Of course, the obligation for individuals to collectively help the vulnerable by realizing herd immunity will not obtain in all public health circumstances. For instance, public health providers may have a record of offering bad service in terms of standards of effectiveness, safety, or transparency [33: 3125]. The moral requirement for individuals to be vaccinated, we contend, can be made only in the presence of reliable medical service, including the administration of safe vaccines.

We want to emphasize two key features of our claim. The first is that any request for exemption for not contributing to herd immunity, as hinted, must be backed by well-established medical reasons, and not by religious or lifestyle beliefs. ${ }^{7}$ The grounding of our case for vaccination in considerations of harm should easily show why personal beliefs or their significance for holders of comprehensive views should be insufficient to secure an exemption. In this paper we will assume that, standardly, individuals are not, or should not be allowed to act upon their religious or lifestyle beliefs if this entails the risk of significant harm to others (provided the action does not produce a collective benefit that others accept). Since holders of religious and lifestyle beliefs can erode the benefit of herd immunity by refusing to vaccinate, they expose others to risk of significant harm. It is thus impermissible for individuals to dismiss their collective moral obligation to realize herd immunity on the grounds of religious and other lifestyle beliefs. ${ }^{8}$ We later discuss whether they may request exemptions if vaccination rates for infectious diseases are well below or well above herd immunity thresholds. ${ }^{9}$

\footnotetext{
5 Hereinafter, we will understand the obligation to be vaccinated to include the obligation to vaccinate one's children, unless stated otherwise.

6 An example of such erosion is a failure of herd immunity in Japan after 1994, causing excess deaths among the elderly. Reichert et al. show that the deaths were due to a drop in influenza vaccination rates among schoolchildren, which previously produced an indirect effect that protected the elderly [30: 893].

7 We understand lifestyle beliefs to include any non-religious comprehensive beliefs that fail to appeal to any public reasons, i.e., those that could resonate with other members of society.

8 As Flanigan's apt analogy shows, "prohibiting religious citizens from turning themselves and their children into biological weapons is a justifiable policy even if religious citizens cannot see it that way" [16: 18].

${ }^{9}$ For a more elaborate account concerning religious exemptions for vaccination, see [28, 29].
} 
Second, here we specifically advocate a moral, and not a legal harm-based obligation to undergo vaccination. By this, we do not suggest that harm brought about by non-vaccination can never be sufficient for establishing a program of mandatory vaccination; if anything, considerations of harm are often thought to be the most obvious driver for instituting coercive policies [23]. Instead, we mean to say that, first, considerations of causing and preventing serious harm should in themselves strongly motivate individuals in their moral behavior. Individuals should not require the threat of having their wrists slapped by the state in order for them and their children to undergo vaccination. If this is, however, insufficient to prompt individuals, legal means may be considered, as 'Plan B', to ensure that the vulnerable are protected, although these would possibly have to be weighed against other important considerations, such as individual liberty and social trust. Second, even if the legal means are justified, governments may find it difficult to come up with appropriate regulation, and in a timely fashion. Such is the current state of the COVID-19 pandemic. In the absence of an established legal framework for vaccination against COVID-19, many individuals might be asking themselves whether they should vaccinate in light of some moral obligation. Our considerations here offer critical guidance for their decisions.

We now turn to showing why the individual obligation to undergo vaccination, if solely driven by realization and preservation of herd immunity, may significantly vary in strength given vaccination coverages in a population. To fully comprehend the effect of herd immunity and of each individual vaccination that contributes to it, we should examine how individual contributions relate to the herd immunity threshold. Jonathan Glover offers a broad distinction between (a) an absolute threshold, where an effect is produced only when the threshold is reached, and (b) a discrimination threshold, where every single increment produces an effect, but such that is too small to be noticed on a large scale [19: 127]. Herd immunity, we find, fits neither of these two categories neatly. Instead, the benefit of each vaccination to the non-vaccinated rises as we approach the herd immunity threshold. In other words, the value of increments peaks at the threshold for that particular infectious disease. This is because individual vaccinations, as we approach the threshold, close off the remaining 'routes of disease' that still threaten the non-vaccinated. Well below the threshold, on the other hand, individual vaccinations are much less significant in themselves for contributing to the collective effect, although their benefit is not completely non-existent. ${ }^{10}$

Imagine a micro-society of ten people that could come under threat of a dangerous infectious disease, for which a vaccine is well-researched, available, and very effective at reducing transmission. Imagine that individuals $X$ and $Y$ are vulnerable, and cannot be vaccinated, while persons $A-H$ can, and if they do, they will all mount the same positive response to the vaccine. Finally, imagine that $X$ and $Y$ rarely if ever come into contact with each other, and will not infect each other if everyone else undergoes vaccination. The starting point, however, is that no one is vaccinated. If

\footnotetext{
$\overline{10}$ It might be more appropriate to speak of a herd immunity peak, rather than a threshold. Once again, however, we stick with the established terminology.
} 
$A$ undergoes vaccination, this benefits $X$ and $Y$, but not significantly, because they can still contract the disease from seven other persons, $B-H$. In numerical terms, $A$ 's vaccination reduces the probability of infection by $12.5 \%$. If $B$ follows $A$ 's example, the benefit is once again small, but greater than when only $A$ vaccinated, since there are now fewer persons who may infect $X$ and $Y$ as a result of $B$ 's vaccination, i.e., this reduces the probability of infection by $14.3 \%$. Fast forward to the final person vaccinating - $H$. If $H$ vaccinates, assuming all other non-vulnerable persons have vaccinated before him, then all the sources of disease for $X$ and $Y$ will have been cut off, and the reduction of the probability of infection will have reached $100 \%$. Thus, $H$ 's vaccination is most significant to $X$ 's and $Y$ 's benefit; although persons $A-G$ have diminished the risk of infection in the micro-society, it is $H$ 's vaccination that puts the stamp on deterring the threat to $X$ and $Y$, and, we believe, produces the strongest moral obligation.

Strictly from the perspective of herd immunity, we believe that the strength of an obligation to vaccinate for the sake of the vulnerable should track the benefit that the single vaccination produces for them. Since the individual benefit of $H$ 's vaccination to $X$ and $Y$ is significantly greater than that of $A, H$ seems to have a stronger obligation to vaccinate than $A$. Marcel Verweij hints at this kind of reasoning, stating that "if most people forgo vaccination against influenza, the effects on public health of my choice for vaccination will become negligible [...] (I)f non-compliance is common, my obligation to contribute to prevention will weaken or even fade away" [34: 329-330]. In a similar way, Derek Parfit has once claimed that individuals should contribute to some collective benefit only if they believed that enough of their peers will act in the same way [26: 77]. If there is no coordination among those potentially undergoing vaccination, and there are few contributors, then from the perspective of realizing herd immunity individuals have a weak moral obligation to vaccinate. ${ }^{11}$

Above the herd immunity threshold, some believe that the individual obligation to vaccinate evaporates completely. Angus Dawson believes that once the herd immunity threshold has been reached, further individual vaccinations produce no additional benefit (and may, in fact, incur a cost), and should therefore not be obligatory [12: 171-177]. Roland Pierik claims that, since herd immunity is sufficient to protect public health, some exemptions to vaccination could be justifiable, if a practicable and justifiable exemption system could be devised [28: 226-228]. Thus, even if we maintain that individuals are obligated to vaccinate just above the threshold, in order to keep the population 'above water', current harm considerations expressed through the value of herd immunity do not explain why individuals should vaccinate well above the herd immunity threshold.

We turn to explicating two compelling arguments grounded in harm that complement the obligation to realize herd immunity. We start from the obligation to vaccinate below the threshold.

\footnotetext{
11 We are making a harm-based claim here. Alternatively, a fairness-based claim could be made that the individual is not obligated to contribute because others are not doing their fair share. We discuss fairnessbased arguments in the third section.
} 


\section{The Obligation Below the Threshold}

The argument that obligations to vaccinate are strongest around the herd immunity threshold leaves us with a counterintuitive implication. If there are a significant number of non-vaccinators who spurn their obligation to contribute to herd immunity, thus moving us further away from the herd immunity threshold, the obligation to vaccinate becomes weaker for everyone. The intuition should rather be, it seems, that if there was a concerted effort by a non-vaccinating group to lower the vaccination rates in a population, the obligation to vaccinate should become stronger for others.

In this section, we argue that aside from the obligation to contribute to a population-level effect in order to minimize the risk of harm to the vulnerable, we should also make sure that we ourselves do not cause significant harm to those around us, provided this comes at no significant cost to us. This obligation is strengthened, we contend, the lower vaccination rates are, because there will then be more of those susceptible to disease, and consequently, we will be more likely to transmit deadly pathogens to others. In order to argue for a stable harm-based obligation to vaccinate below the herd immunity threshold, we must accept, or so we claim, both the collective obligation to establish herd immunity elaborated in the previous section, and the obligation that we ourselves not harm others, which we elaborate here.

The basic argument is simple. Although my contribution to herd immunity might be insignificant, given inadequate contributions by others, my infectiousness could still be decisive in whether a particular person will become dangerously infected, possibly causing that person significant harm [17: 551]. Since it would be wrong for people to expose others to risk of significant harm, they have the obligation to vaccinate, thus curbing their (prospective) infectiousness better than they would otherwise be able to. Admittedly, the chances that we ourselves would transmit infection are small in a population where virtually everyone is just as much a threat. But as Jessica Flanigan says, this does not entitle non-vaccinators "to harm others, despite the fact that the risk of harm is of low-probability, their victims are unlikely to identify them, and they do not intend to injure their victims" [16: 8]. Flanigan illustrates this point neatly with her comparison between not vaccinating and firing a gun into the air and thereby endangering innocent by-standers. The refusal to vaccinate, she claims, constitutes the same kind of potentially harmful reckless conduct, and exposes others to risk of harm in a morally analogous way [16: 7].

The obligation that we ourselves not expose others to harm is further strengthened, we claim, by the increased numbers of those that we are exposing. Because most individuals are, by hypothesis, neglecting their obligation to vaccinate, there will be more of those who might be struck by our proverbial bullets of nonvaccination. The significance of non-vaccination risk is thus increased, it seems, as "the pool of people who are exposed to the risks of transmission becomes 
greater" [16: 12]. ${ }^{12}$ If this is correct, and if our sense of obligation should track the numbers of those who we might endanger, then the moral obligation to vaccinate should become stronger the lower vaccination rates are. This explains why we might believe that there are specially placed individuals in society, like the staff in nursing homes [34: 326] and health care workers [2], who should have a particularly strong obligation to vaccinate due to the number of vulnerable people with whom they come into contact.

This is not to suggest that exposing a single vulnerable person to risk of serious harm represents only a minimal moral transgression. We only contend that our moral sentiment seems rightly sensitive to the prospect of harming many, compared to the prospect of harming only few. It would be morally negligent to shoot bullets into an area containing a single person, but it seems downright atrocious to open fire in a densely populated area. Still, both acts should be considered morally wrong. ${ }^{13}$

It could be argued that Flanigan's gun-firing example does not accurately illustrate the manner in which individuals expose each other to risk of harm when vaccination rates are low. If not being vaccinated is sufficient for individuals to be as negligent as those who fire their guns into the air, then the circumstances of low vaccination rates are more akin to a gun-firing free-for-all, in which the majority of the population participates. Perhaps not vaccinating is indeed on a par with firing a gun into the air and exposing others to risk of harm, but these others expose us to risk of harm in exactly the same way, and, assuming they have access to vaccination and are refusing it, should hardly be characterized as innocent by-standers. At the very least, they knowingly and willingly expose themselves to risk of harm. Revising the gun-firing analogy in this way prompts two objections that bring our obligation well below the threshold into question.

First, what do we owe those who have knowingly and willingly placed themselves under risk of serious harm? While it seems beyond doubt that we have an obligation to protect the vulnerable (those listed in the first section), others have become vulnerable of their own accord, and have, in addition, made themselves threats. Why should we have to undergo vaccination for their sake? Knowingly and willingly exposing oneself to harm of infection should, arguably, not add to the obligation set that others need to abide by to prevent that harm.

Still, this hardly seems sufficient to waive already existing obligations within that set, which certainly includes adopting low-cost measures of prevention. Imagine that a jaywalker is crossing the road that you are driving on. If you do not significantly reduce your speed, which is, presumably, within permitted limits,

\footnotetext{
${ }^{12}$ It should be noted that the extent of the risk is also determined by other factors, such as density or size of the population that is being endangered; it would be desirable if feelings of moral obligation were sensitive to these factors as well. We will treat them here as background factors. Unless a population is particularly small and sparse, agents should assume that an increase in the number of the non-vaccinated makes the risk of their own non-vaccination more significant.

13 Note that the numbers factor should also bear on our considerations in the sense that we could be decisive in the chain of transmissions that reaches the vulnerable who cannot be vaccinated for medical reasons. The more non-vaccinated individuals there are, the more routes of disease there are for "our" pathogens to potentially reach the vulnerable.
} 
you will risk hitting the jaywalker and significantly harming him. Do you have an obligation to hit the brakes and reduce your speed? We believe most would share the intuition that you do. Note that carrying this obligation out even comes with a small risk to your own safety (hitting the brakes could cause a serious traffic accident); yet, we believe the intuition that we are obliged to hit the brakes would persist.

It could be maintained that an obligation to adopt minimal measures of harm prevention does not obviously translate into an obligation to vaccinate, since there are other less demanding measures that could be taken to prevent the transmission of pathogens (like staying at home, maintaining a distance from others, or wearing a face mask). The controversial question is what constitutes this 'bare minimum' in harm prevention. Does it require less than vaccination? Supportive of vaccination as this bare minimum is the fact that, with many diseases, individuals could be asymptomatic carriers and represent viable threats without knowing it. Adopting other measures might only be sufficient to prevent harm if they are undertaken most of the time.

Second, in a gun-firing free-for-all, any person could be harmed by any number of shooters. In circumstances of low vaccination rates, it could be argued that my vaccination will prevent me from infecting another person, but will ultimately not make a significant difference to whether that person is infected. If persons are exposed to the extent that they will be infected anyway, the objection goes, then we should not be burdened by the obligation to vaccinate. Our knee-jerk reply to this objection is that such predictive certainty is unconvincing in considerations of public health, and for all individuals. But let's imagine for the sake of argument that the prediction holds water. Even then, some authors claim, not vaccinating would be wrongful. Jason Brennan has argued that we should abide by the 'clean hands principle', according to which there is a "moral obligation not to participate in collectively harmful activities", even if the outcome is overdetermined [5: 40]. Brennan uses the example of a firing squad, in which:

A band of 10 sharpshooters is about to kill an innocent child. They have been trained to shoot in such a way that each shot will hit the child at the same time, and each shot would be fatal on its own. You can't stop them from killing the child. They ask you if you'd like to join in and take the $11^{\text {th }}$ shot [5: 40].

Brennan argues that joining in with the sharpshooters violates the clean hands principle. Unlike the harm-based arguments we have so far defended, this principle points to a wrongness that is not sensitive to vaccination rates or considerations of prevention on the population level. Still, the principle would be sensitive to the consideration that, well below the threshold, there is a great number of those exposed to risk of harm. By not vaccinating, an individual could participate in the harm of many, thereby violating the clean hands principle for each exposed individual. Thus, if we are convinced by the clean hands principle, the numbers of those susceptible to harm should still be a weighty consideration. Therefore, even if vaccination makes no difference to those around us (which is at best dubious as a general prediction), 
it could still constitute a violation of a moral rule that is more likely and frequently broken well below the herd immunity threshold. ${ }^{14}$

Let us take stock. In this and the previous section, we have presented the first two harm-based arguments for a moral obligation to vaccinate. Most importantly, we argued that the two arguments run in opposite directions in terms of the strength of the obligation that they establish, given the different levels of vaccination coverage. Whereas the herd immunity argument establishes that the obligation becomes stronger as we draw closer to the herd immunity threshold, but is somewhat weak well below the threshold, the argument that we not harm others ourselves generates the strongest obligation at the lowest levels of vaccination coverage. The two arguments are appealed to at different levels of vaccination coverage, which is why both are required for a stable harm-based moral obligation to vaccinate. But is the obligation stable even beyond the herd immunity threshold? We turn to this in the following section.

\section{The Obligation Above the Threshold}

In the previous two sections, we have spelled out two arguments grounding an individual obligation to vaccinate when rates are close to the herd immunity threshold (on either side) and well below it. If individuals discharged this obligation up to the point of reaching herd immunity thresholds for all mentioned infectious diseases, an important milestone for public health would already be reached. But would the obligation for individuals persist if herd immunity is secured? As stated in the first section, many individuals will want to avoid vaccination for religious and other lifestyle beliefs. We claim that for considerations of feasibility, which are relevant in virtually any modern social setting, the obligation to vaccinate should persist. In claiming this, we rely strictly on harm-based considerations.

Accounts centered on harm have already been flagged, mainly by their own proponents, for their problems with grounding the obligation to vaccinate once the good of herd immunity has been obtained. As we mentioned, Dawson argues against such an obligation because vaccinating above the herd immunity threshold produces no additional benefit to others, since herd immunity will have already minimized potential risks of harm; in fact, individuals who vaccinate in these circumstances are exposed to unnecessary risk given vaccination's possible adverse effects [12: 171-177]. In other words, why expose a person to risk of vaccination if she is already protected by herd immunity? In a similar vein, Pierik believes that high vaccination rates might allow for exemptions from vaccination [28: 226]. ${ }^{15}$ However, both Dawson and Pierik stress that there are important pragmatic reasons to maintain vaccination rates as high as possible. Here, we offer two of what we regard

\footnotetext{
${ }^{14}$ We do not discuss the principle at length here, mostly because we are not convinced by the assumption that single vaccinations never make a difference well below the threshold.

15 Similarly, Brennan holds that vaccination should not be mandatory if vaccination rates are sufficiently high [5: 37].
} 
to be the main pragmatic reasons that lend support to maintaining the obligation to vaccinate above the threshold. These weighty reasons arise in circumstances that, we hold, are ubiquitous even in the most advanced, currently feasible public health settings.

Before turning to these reasons, we should note that arguments in favor of the obligation to vaccinate have also been forwarded on fairness-based accounts. Most recently, Alberto Giubilini has argued that any individual who is able to reasonably bear her fair share of the burden in realizing herd immunity has an obligation to do so, regardless of the impact that her contribution makes to the collective outcome [18: 50]. Deciding not to contribute our part in fulfilling a collective obligation means treating other obligation holders unfairly [17: 555]. A purported strength of a fairness-based account is that it is supposedly more successful than the harm-based account at explaining why the obligation should persist beyond the herd immunity threshold. If individuals do not have medical reasons for non-vaccination, but have failed to take up their reasonable share of the collective burden, then they are treating those who have contributed to the collective good of herd immunity unfairly, even if herd immunity has already been realized. Thus, the fairness-based account more easily accommodates the intuition that there is something wrong with freeriding on herd immunity.

The soundness of this moral implication hardly seems controversial. Yet, our focus here remains on the harm-based rationale, for two reasons. We want to suggest that fairness considerations are either merely complementary to considerations of harm, or merely secondary to them.

Let's take each possibility in turn. As we will show in this section, on a harmbased account, individuals are advised to vaccinate above the herd immunity threshold as a final precaution against herd immunity breaking down and against individuals themselves exposing others to risk of serious harm. Insofar, the argument for vaccinating above the threshold is supplementary to our two previous (and more foundational) arguments in favor of vaccination, in circumstances below and around the threshold. A fairness-based account, on the other hand, successfully justifies the obligation around and above the threshold, but makes a much weaker case for it well below the threshold. If hardly anyone undergoes vaccination in a population, then it is hard to see why an individual would be treating others unfairly by not vaccinating. Giubilini et al. themselves acknowledge that if a fairness-based obligation existed in such circumstances, such an obligation would be weak, "and indeed it would be the weaker, the higher the number of people around me who fail to make their contribution" [17: 558]. Thus, using fairness as a standalone principle runs into difficulties with getting the obligation to vaccinate off the ground, when vaccination rates are extremely low. But Giubilini et al. add that fairness considerations can be replaced in such circumstances by considerations of harm, since individuals would retain the obligation "to be vaccinated in order to minimize the risk of harming others" [17: 558]. Therefore, the fairness account fails at grounding the obligation below the threshold, and requires an alternative justification to cover a particularly significant blind spot, for which harm-based considerations are particularly suitable. The harm-based account, on the other hand, establishes the moral obligation to vaccinate, based 
on the two arguments we have presented in the previous two sections, from low vaccination rates to at least the point of realizing herd immunity. In this sense, the fairness account may be merely complementary to considerations of harm, since the latter seem to be more foundational in establishing the obligation.

But the fairness complement is required, we believe, only if considerations of harm cannot by themselves justify the obligation to vaccinate in all circumstances, which this section will try to argue against. If harm can justify the obligation in all circumstances, then, we believe, fairness considerations, although sound, become merely secondary to them. Although the fairness argument successfully highlights the injustice of not doing your fair share and free-riding on the contributions of others, these contributions concern the establishment of herd immunity, the purpose of which is to minimize harm. Surely, individuals should care about not treating others unfairly, but a more pertinent consideration in matters of infection is whether others will be exposed to risk of possibly deadly harm as a result of our inaction. Insofar, as long as harm can justify the obligation on all sides of the herd immunity threshold, it should be a sufficient and more primary justification for the obligation to vaccinate. Giubilini himself states that this might be the limit of the fairness consideration:

We can think of fairness as a subordinate or secondary goal of vaccination policies: we do not enforce vaccination policies in order to promote fairness, but once we decide to enforce vaccination policies in order to realize herd immunity and prevent harm, fairness does become one of the goals of these policies, because herd immunity should be realized fairly [18: 108; emphasis in original].

Let us now turn to the pragmatic reasons for vaccination above the threshold. They point to the feasibility constraints in establishing the conditions of minimal harm from infection, and serve as a precaution by buttressing herd immunity. We will mention two such reasons here.

First, even in the most modern public health settings, vaccination rates are prone to changes, which are often difficult to track in a timely and precise fashion. In fact, one aspect of the modern setting-population movements-arguably exacerbates these issues. Because individuals are now able to effortlessly travel from one country to another [17: 557], and change one's place of residence much more easily than only a few decades ago, public health systems are facing greater challenges to make reliable estimations about vaccination coverages. For instance, in cities (like Dubrovnik) and countries that host a significant number of tourists, this will be a near impossible task, at least during the tourist season. Attempting to protect the vulnerable may thus require even higher vaccination rates, under the precautionary assumption that the individuals coming into contact with the population are not vaccinated. In addition, individuals should be expected to vaccinate not only to protect the vulnerable members of their own population, but also of other populations, when they travel or migrate. There are, of course, further reasons why vaccination rates are sometimes unstable, and their monitoring unreliable. Fine et al. state that statistics may be inaccurate (or even falsified), and that vaccination is sometimes poorly administered or taken outside the recommended schedule [15: 914]. Or consider that 
vaccination rates have a standard tendency to drop, potentially below herd immunity levels, when children are born and vaccinated persons die.

The non-vaccinated person may thus face epistemic problems if she is attempting to assess the strength of her obligation by looking at immediate vaccination rates. These rates are both sufficiently unstable, and their tracking too delayed for individuals to make informed assessments about the exact strength of their obligations. Recall that for most of these infectious diseases, herd immunity thresholds are over $90 \%$, and it may represent a significant difference to the obligations of individuals whether the rates are one or two percentage points up or down. Because reliable information is often unavailable, individuals ought to vaccinate as a matter of precaution. ${ }^{16}$

Second, we mentioned earlier that herd immunity estimations are made under the assumption of a "homogenously mixed" population. This assumption is often unwarranted, due to "age-related, genetic, geographical, social, and behavioural factors" [1: 643]. Non-vaccinated individuals are often clustered together, for example, in schools and communities [29: 391], making them more vulnerable to outbreaks [15: 914]. These population clusters are particularly formed around shared religious beliefs, i.e., among individuals who are granted religious exemptions from vaccination $([14,24])$. Since these non-vaccinated individuals tend to interact with one another more so than with others, they find themselves at increased risk of harm [15: 914]. One recent finding strongly implicating this point concerns Jewish and Mennonite communities in New York, who accounted for over $75 \%$ of all measles cases in the US in 2019 [8]. When population clusters are present, the population might require a higher vaccination rate in order to be protected ([1: 643, 28: 228]). This effectively places a significant feasibility constraint on the normative possibility for individuals to reject vaccination on religious and lifestyle grounds above the threshold.

Holders of these religious beliefs might object that putting pressures on them to vaccinate is objectionably paternalistic to them and to other members of their religious communities. As part of a population cluster, they primarily place those individuals under risk of harm who would be willing to accept such risks anyway. In addition, the content of their beliefs makes vaccination particularly costly to them, and their peers would never require them to compromise their way of life by vaccinating. In short, the idea is that pressuring the religious community into adopting

\footnotetext{
16 We have to make an important concession here. Since arguments in favor of vaccinating above the herd immunity threshold represent precautionary considerations meant for buttressing herd immunity, they will be stronger the higher herd immunity thresholds are for particular diseases. This is because the higher herd immunity thresholds are, the less "room" there is above the threshold, and consequently, the more there is reason for precaution. Insofar, the harm-based argument for vaccinating above the threshold is stronger for diseases like measles (herd immunity threshold of 92-94\%) and pertussis (also 92-94\%) than for mumps $(75-86 \%)$ or polio $(80-86 \%)$. This is not to say that precautionary considerations do not apply to diseases with herd immunity thresholds below, say, $90 \%$, but it might be feasible for some diseases that precautions beyond a certain point are no longer necessary. Even so, in such cases, fairnessbased considerations might complement harm-based ones. We thank Abhishek Mishra for raising this concern to us.
} 
the obligation to vaccinate, in order to minimize infection among them, disregards what members of this community consider to be good for them.

While it does seem reasonable for members of population clusters to agree to greater risk of harm, they should not be permitted "to put their children at avoidable risk of death and suffering" [29: 382]. Children in population clusters (or at least a significant portion of them) cannot provide informed consent to risks of harm brought about by non-vaccination. Given that parents will not be able to avoid coming into potentially infectious contact with their own children, these risks cannot be isolated only to those who would consent to them. Adults within the population clusters could argue that they will vaccinate their children, while remaining unvaccinated themselves. This will indeed protect most children within the cluster, but some will remain under significant risk of infection. This is because some children are either too young to be vaccinated, or they belong to one of the two other vulnerable groups. ${ }^{17}$ Given the greater incidence of infectious disease in population clusters, vulnerable children will inescapably be exposed to considerable risks. An obligation to vaccinate should thus persist, even for adults within population clusters.

Finally, some authors have suggested that requirements for vaccination in a population could be arranged via a lottery ([5: 37, 28: 235]). The purpose of the lottery would be to provide exemptions for a small minority, but with an algorithm in place that would ensure homogeneity. Typically, we would conceive of such a suggestion to regulate mandatory vaccination, but it is not outside the realm of possibility for it to strictly coordinate our moral obligations to vaccinate. If such a lottery scheme were feasible, then the harm-based argument for vaccinating above the threshold would lose force, and our claim that the moral obligation is stable on both sides of the threshold would be compromised. However, the lottery proposal runs into its own feasibility problems. For one, the lottery would have to be frequently repeated given the instability of vaccination rates that we described. Additionally, if the purpose of the lottery is only to help us assign a moral (and not a legal) obligation, the program would somehow have to compensate for the possibility of imperfect compliance. This would once again likely be accomplished by drawing fewer lots for exemption, thereby possibly assigning some obligations above the threshold. In short, the lottery proposal could establish some variance in assuming the obligation, but it remains to be seen whether this can be worked out in practice. ${ }^{18}$

\footnotetext{
17 Consider, also, that vaccination is not a guarantee against infection (the measles vaccine, for instance, is only $93 \%$ effective after one dose, and $97 \%$ effective after two doses [9]).

18 We thank Luca Malatesti for bringing the lottery objection to our attention. Interestingly, if the lottery scheme could achieve the disappearance of the obligation above the threshold on a harm-based account, it would likely do so on a fairness-based account as well. Assume that everyone in the population enters the lottery, i.e., that no one is trying to avoid it, and that everyone intends to undergo vaccination if they are not drawn. Assume also that there is no foul play in the lottery itself. It could be argued that the person who is drawn, and is thereby licensed to avoid vaccination, is not treating others unfairly when she does not vaccinate. She has accepted the same odds and terms as everyone else. Unless we believe there is something unfair about these terms, which is not obvious in the least, there seems little reason to reject that the outcome of the lottery is fair.
} 


\section{Conclusion}

In this paper, we offered three harm-based arguments in favor of a strong individual moral obligation to undergo vaccination. These arguments, we believe, show our obligation to be strong even when the population is well below or above a herd immunity threshold. First, we argued that individuals should participate in the collective effort of establishing herd immunity, thereby minimizing the spread of infection. Second, contrary to the claim that our obligation to vaccinate wears off when the population is well below the herd immunity threshold, we argued that it is in fact strengthened the lower vaccination rates are, given the increased numbers of those who we might put in harm's way through our individual infectiousness. Third, we provided pragmatic reasons for the claim that the moral obligation persists when the population has already established herd immunity, that is, once the population is well above the herd immunity threshold. These pragmatic reasons, we contend, are grounded in the feasibility constraints of establishing the conditions of minimal harm resulting from infections. Vaccination rates are difficult to track and are susceptible to changes due to population movements and shifting population demographics. Owing to the related epistemic issues pertaining to vaccination rates, the individual moral obligation to undergo vaccination persists in the form of a precautionary measure.

Acknowledgement We would like to thank Abhishek Mishra, Aleksandar Simić, Luca Malatesti, Ivana Munitić, Tvrtko Jolić, Elvio Baccarini, and Peter Young for providing us with valuable feedback throughout the writing process. We also thank two anonymous reviewers for their useful comments.

Funding Viktor Ivanković is supported by the Croatian Science Foundation (Grant Reference Number: HRZZ-UIP-2017-05-4308), as part of the 'Harm, Intentions and Responsibility' (HIRe) project. Lovro Savić is supported by Wellcome Trust. This research was funded in whole, or in part, by the Wellcome Trust [Grant number 212764/Z/18/Z]. For the purpose of open access, the author has applied a CC BY public copyright licence to any Author Accepted Manuscript version arising from this submission.

\section{Declarations}

Conflict of interest The authors declare that they have no conflict of interest.

\section{References}

1. Anderson, R. M., \& May, R. M. (1990). Immunisation and herd immunity. The Lancet, 335(8690), 641-645.

2. Anikeeva, O., Braunack-Mayer, A., \& Rogers, W. (2009). Requiring influenza vaccination for health care workers. American Journal of Public Health, 99(1), 24-29.

3. Antona, D., et al. (2013). Measles elimination efforts and 2008-2011 outbreak, France. Emerging Infectious Diseases, 19(3), 357-364.

4. Battin, M. P., et al. (2009). The patient as victim and vector: Ethics and infectious disease. Oxford University Press.

5. Brennan, J. (2018). A libertarian case for mandatory vaccination. Journal of Medical Ethics, 44(1), 37-43.

6. CDC (2019a). Guillain Barré Syndrome. Retrieved 15 January 2020 from https://www.cdc.gov/vaccinesaf ety/concerns/guillain-barre-syndrome.html

7. CDC (2019b). Measles, Mumps, and Rubella (MMR) Vaccination: What everyone should know. Retrieved 27 March 2020 from https://www.cdc.gov/vaccines/vpd/mmr/public/index.html 
8. CDC (2019c). National Update on Measles Cases and Outbreaks - United States, January 1-October 1, 2019. Retrieved 30 March 2020 from https://www.cdc.gov/mmwr/volumes/68/wr/mm6840e2.htm

9. CDC (2019d). Vaccine for Measles. Retrieved 4 April 2020 from https://www.cdc.gov/measles/vacci nation.html

10. CDC (2020). Recommended Adult Immunization Schedule for ages 19 years or older, United States, 2020. Retrieved 4 February 2020 from http://www.cdc.gov/vaccines/schedules/hcp/imz/adult.html

11. Cox, N. J., \& Subbarao, K. (2000). Global epidemiology of influenza: Past and present. Annual Review of Medicine, 51, 407-421.

12. Dawson, A. (2007). Herd protection as a public good: Vaccination and our obligations to others. In A. A. Dawson \& M. Verweij (Eds.), Ethics, prevention and public health (pp. 160-178). Clarendon Press.

13. de Rugy, V., \& Peña, C. V. (2002). Responding to the threat of smallpox bioterrorism: An ounce of prevention is best approach. Policy Analysis, 434, 1-16.

14. Feikin, D. R., et al. (2000). Individual and community risks of measles and pertussis associated with personal exemptions to immunization. JAMA The Journal of the American Medical Association, 284(24), 3145-3150.

15. Fine, P., Eames, K., \& Heymann, D. L. (2011). "Herd Immunity": A rough guide. Clinical Infectious Diseases, 52(7), 911-916.

16. Flanigan, J. (2014). A defense of compulsory vaccination. HEC Forum, 26, 5-25.

17. Giubilini, A., Douglas, T., \& Savulescu, J. (2018). The moral obligation to be vaccinated: Utilitarianism, contractualism, and collective easy rescue. Medicine, Health Care and Philosophy, 21, 547-560.

18. Giubilini, A. (2019). The ethics of vaccination. Palgrave MacMillan.

19. Glover, J. (1986). It Makes No Difference Whether or Not I Do It. In P. Singer (Ed.), Applied ethics (pp. 125-144). Oxford University Press.

20. Hansson, S. O. (2011). Risk. The Stanford Encyclopedia of Philosophy. Retrieved 15 January 2020 from https://plato.stanford.edu/entries/risk/.

21. Looker, C., \& Kelly, H. (2011). No-fault compensation following adverse events attributed to vaccination: A review of international programmes. Bulletin of the World Health Organization, 89, 371-378.

22. Malone, K. M., \& Hinman, A. R. (2007). Vaccination mandates: The public health imperative and individual rights. In R. A. Goodman, R. E. Hoffman, W. Lopez, G. W. Matthews, M. Rothstein, \& K. Foster (Eds.), Law in public health practice (pp. 262-284). Oxford University Press.

23. Mill, J. S. ([1859] 1974). On Liberty. Hammondsworth: Penguin.

24. Omer, S. B., et al. (2008). Geographic clustering of nonmedical exemptions to school immunization requirements and associations with geographic clustering of pertussis. American Journal of Epidemiology, 168(12), 1389-1396.

25. Oxford Vaccine Group (2015). Measles. Retrieved 13 February 2020 from http://vk.ovg.ox.ac.uk/measl es

26. Parfit, D. (1984). Reasons and persons. Oxford University Press.

27. Paul, Y. (2004). Letter: Herd immunity and herd protection. Vaccine, 22(3-4), 301-302.

28. Pierik, R. (2017). On religious and secular exemptions: A case study of childhood vaccination waivers. Ethnicities, 17(2), 220-241.

29. Pierik, R. (2018). Mandatory vaccination: An unqualified defence. Journal of Applied Philosophy, 35(2), 381-398.

30. Reichert, T. A., et al. (2001). The Japanese experience with vaccinating schoolchildren against influenza. The New England Journal of Medicine, 344, 889-896.

31 Tomljenovic, M., et al. (2020). Measles outbreak in Dubrovnik-Neretva County, Croatia, May to June 2018. Eurosurveillance. https://doi.org/10.2807/1560-7917.ES.2020.25.7.1900434

32. Ulmer, J. B., \& Liu, M. A. (2002). Ethical issues for vaccines and immunization. Nature Reviews Immunology, 2, 291-296.

33. Verweij, M., \& Dawson, A. (2004). Ethical principles for collective immunization programmes. Vaccine, 22(23-24), 3122-3126.

34. Verweij, M. (2005). Obligatory precautions against infection. Bioethics, 19(4), 323-335.

Publisher's Note Springer Nature remains neutral with regard to jurisdictional claims in published maps and institutional affiliations. 